\title{
La dialettica del mito. Leucò, ovvero le "Operette morali" di Cesare Pavese
}

\author{
FRANCESCO MUZZIOLI \\ Università La Sapienza, Roma \\ francescomuzzioli@uniroma1.it
}

\section{Riassunto}

Il saggio analizza il componimento di Pavese: Atlantic oil, appartenente alla raccolta di Lavorare stanca, allo scopo di dimostrare la doppia prospettiva semantica, sociale e personale, di questo testo. In esso interagiscono ricche e polisemiche presenze immaginarie, le quali, studiate alla luce della teoria del simbolo e dell'allegoria di base benjaminiana, ci rivelano ancora una volta i paradossi della scrittura pavesiana. Questa perviene ad una rappresentazione solcata dalla propria crisi, cioè dalla sua più evidente modernità.

Parole chiave: Poesia, Pavese, duplicità, paradossi, Atlantic oil, Lavorare stanca, Leucò, simbolo, crisi, modernità.

The dialectics of myth. Leucò or the «Operette morali» of Cesare Pavese

\begin{abstract}
The essay analizes the poem of Pavese Atlantic oil, included in his compilation Lavorare stanca, trying to demonstrate the double semantic perspective, social and individual, of this text. In this poem intermingles rich and polysemic imaginary presences, which are studied according to the benjaminian symbol and allegorical theory. This analysis reveals once more the paradoxes of pavesian writing, that stems out from his own crisis, that is, from his evident modernity.
\end{abstract}

Key words: Poetry, Pavese, duplicity, paradox, Atlantic oil, Lavorare stanca, Leucò, symbol, crisis, modernity.

Muzzioli, Francesco. 2011.La dialettica del mito. Leucò, ovvero le «Operette morali» di Cesare Pavese. Cuadernos de Filología Italiana, $\mathrm{n}^{\mathrm{o}}$ extraordinario: págs. 269-281.

Non sarebbe difficile riscontrare il segno della "duplicità" alla base del percorso letterario di Cesare Pavese. La sua scrittura parte sul terreno della poesia ma vi prende subito la piega del racconto, quindi deborda pressoché definitivamente verso 
l'ambito narrativo. Pavese è fin dall'inizio un autore di impegno politico-sociale, ma ciò che lo spinge a scrivere è inequivocabilmente una "ferita" esistenziale e psicologica, attorno alla quale si ostina a scavare testo dopo testo. È senza dubbio un realista, interessato al livello concreto e materiale sia nella sfera pratica che in quella del corpo, ha una propensione per il parlato dialettale degli strati bassi della popolazione (contadini, operai, sradicati urbani), eppure è un autore e addirittura un teorico del mito, cioè di un «universale umano» colto attraversando la storia. Fa sua la bandiera della maturità (l' «arte di maturare»), tuttavia si propone il ritorno all'infanzia e non solo si presta benissimo a una indagine psicoanalitica, come qualsiasi scrittore in fin dei conti, ma è lui per primo a farsi carico dell'osservazione dell'inconscio. Ciò complica anche l'autocoscienza pavesiana sul tema della tecnica, perché la nozione di «lavoro poetico», che lo accompagna fino dal Mestiere di poeta, deve coabitare con l'accoglimento della ispirazione imponderabile. La stessa biografia, con il suo esito, sottolinea nel tragico una delle chiavi di lettura decisive: eppure la scrittura è venata dall'ironia e il dialogo dalla continua ricerca di battute e risposte spiritose.

L'intenzione di perseguire insieme l'immagine e il racconto, ovvero, di «raccontare immagini», come Pavese dice al termine del percorso di Lavorare stanca ${ }^{1}$, è esattamente il concentrato della duplicità di due direzioni antitetiche, la discorsività e la figuralità. Potremmo dire in altri termini, ormai classici per la teoria della letteratura dopo Jakobson, Lacan e molti altri, metonimia e metafora: e basterà un brevissimo passaggio di analisi del testo per rendersi conto di come proprio queste due istanze, per quanto si intreccino, per quanto collaborino, nel testo pavesiano non siano mai in equilibrio, ma sempre in una sorta di sovrapposizione e coabitazione forzata.

Prendiamo per prova Atlantic oil, il testo che ha per titolo una marca di benzina di allora (è datata 1933) e che configura perfettamente la questione città/campagna, così come la vede Pavese. Città in campagna è il titolo della sezione e l'obiettivo è posto proprio sull'avanzata della città che però ancora non riesce a conquistare la campagna. Certo, la modernizzazione è in arrivo e ce ne avverte il distributore di benzina, una sorta di avamposto dell'invasione delle macchine. Per chi volesse ricostruire il luogo preciso, una minuta allude all' «autos. Chieri-Torino». Ma il progresso è lento, l'Italia rimane ancora agricola, i contadini hanno le bestie (sono «bestie» anche loro, direbbe Pavese), e diffidano delle macchine, il benzinaio (che a quest'altezza temporale si chiama «meccanico», forse perché il distributore è, per necessità, anche un'officina) si trova fuori posto, passano pochi avventori, per la noia non resta che ubriacarsi. Gli elementi invasori del moderno-artificiale vengono accerchiati dall'ambiente campestre e pian piano riassorbiti nella natura. Vediamo, infatti: il meccanico sbronzo è felice buttato in un fosso (Pavese 1973b: 75).

Comincia e già, in questo attacco, il personaggio è come ritornato alla terra $\mathrm{e}$ questo ritorno, subito qui, richiama la morte, il seppellimento. Poco oltre:

${ }^{1}$ Vedi «A proposito di certe poesie non ancora scritte (1940)», in (Pavese (1973b: 137). 
A due passi, nel prato, è rizzato il cartello rosso e nero: chi troppo s'accosti, non riesce più a leggerlo, tanto è largo. A quest'ora è ancor umido di rugiada. La strada, di giorno, lo copre di polvere, come copre i cespugli. Il meccanico, sotto, si stira nel sonno (Pavese 1973b: 75).

È una poesia del lavoro (del resto perfettamente in sincrono con il titolo della raccolta, Lavorare stanca) e di tema polemicamente "basso" rispetto all'iperuranio metafisico dei lirici ermetici. Tuttavia è un lavoro curioso, saltuario, in qualche modo pigro.

I meccanici, un poco intontiti, saranno al mattino sui bidoni, seduti, aspettando un lavoro.

Fa piacere passare il mattino seduto nell'ombra. Qui la puzza degli olii si mesce all'odore di verde, di tabacco e di vino, e il lavoro li viene a trovare sulla porta di casa (Pavese 1973b: 75).

L'avanguardia del moderno diventa un confine poroso, un punto di mescolanza. E andiamo al finale dove le automobili assomigliano ad animali notturni, che si muovono con passi felpati:

Sotto l'alba trascorrono caute e non s'ode rumore se non brezza che passa, e toccata la cima si dileguano nella pianura, affondando nell'ombra (Pavese 1973b: 76).

Ancora un'immagine un po' mortuaria, di inabissamento verso il buio. Ma l'intreccio-scontro di metonimia e metafora è soprattutto in due passi; il primo:

[le macchine] Improvvise alla cima del colle, rallentano un poco, poi si buttano giù dalla curva. Qualcuna si ferma nella polvere, avanti al garage, che la imbeve di litri (Pavese 1973b: 75).

La metonimia è il «garage» divenuto soggetto del dare la benzina, cioè il contenente per il contenuto, che trasmette l'idea dell'alienazione, della sostituzione del lavoro astratto al lavoratore concreto. La metafora invece è il verbo «imbevere» che trasforma l'automobile in qualcosa di naturale, una spugna o il terreno stesso, quando viene innaffiato. Le due figure si sorreggono bene e gli fa corona ulteriore la sineddoche generalizzante «litri», al posto di litri di benzina, che aiuta la trasformazione della benzina in un liquido benefico che irrora. Fin qui c'è una distribuzione delle parti, ma le cose si svolgono con maggiore attrito più avanti. Quando si prospetta, con un'apertura al futuro, la ri-conversione del meccanico in contadino, dove veramente la natura e il passato riprenderebbero i loro poteri. La molla è il matrimonio, quindi la donna:

Tra le coste, c'è sempre una vigna che piace sulle altre:

finirà che il meccanico sposa la vigna che piace 
con la cara ragazza, e uscirà dentro il sole ma a zappare, e verrà tutto nero sul collo e berrà del suo vino, torchiato le sere d'autunno in cantina (Pavese 1973b: 75-76).

Il «meccanico sposa la vigna»: è una metonimia evidentemente («nemmeno in Spagna si potrebbe...»), il meccanico sposerà una ragazza proprietaria o ereditiera della vigna che ha adocchiato. Una metonimia cinica che sottolinea il matrimonio di interesse, tanto più cinica in quanto rara metonimia in praesentia (e invece di dire «sposa la ragazza con la vigna» sposa la vigna «con la cara ragazza», come fosse un optional). Eppure, eppure, che sotto questa lectio facilior ci sia una lectio difficilior, dove la vigna non è una metonimia ma una metafora, non sarebbe poi tanto strano (e basterebbe l'ultimo Pavese: «Sei la vigna $»^{2} \ldots$.. . La figura, che funziona alla superficie come forma di ironia (lo spostamento metonimico dice la pura e semplice verità: l'obiettivo, l'oggetto amato, è la vigna), funziona in profondità come una catena fatale: sposa la donna, ma la donna è una vigna, la vigna è natura, e la natura è la morte.

Proprio questo sottofondo rivelato dalla lettura metaforica Pavese proverà ad estrarlo e ad esporlo attraverso la teoria del simbolo. Entrando nella "jungla" (per dirla con Eco) delle teorie del simbolo: in alleanza con la tradizione romantico-idealista - quella, per intenderci, che parte da Goethe e che pone il simbolo all'apice dell'esperienza estetica, come forma di fusione e trasfigurazione del significato nel significante - anche Pavese sottolinea l'approccio intuitivo e il particolare investimento. Il simbolo è un rappresentante contraddistinto dal forte senso di pienezza che trasmette; è un'entità magicamente rivestita di potere comunicativo, che mette in comunicazione con un senso sopraordinato. La teoria di Pavese, accennata nel diario, troverà sviluppo e articolazione soprattutto negli scritti collegati ai racconti di Feria d'agosto. Lo scritto più completo e chiaro, in proposito è Del mito, del simbolo e d'altro (siamo nel 1943-44) in cui l'evidenza intuitiva viene colta nell'apparizione di un luogo particolarmente intenso, un «luogo unico», che si propone perciò come matrice di senso (contiene tanti altri luoghi ed eventi della vita) e come fondatore dell'esperienza (si tratta di "riconoscerlo" tra tanti):

Quest'unicità del luogo è parte, del resto, di quella generale unicità del gesto e dell'evento, assoluti e quindi simbolici, che costituisce l'agire mitico. Una definizione non retorica di questo sarebbe: fare una cosa una volta per tutte, che perciò si riempie di significati e sempre se ne andrà riempiendo, in grazia appunto alla sua fissità non più realistica. Nella realtà naturale nessun gesto e nessun luogo vale più di un altro. Nell'agire mitico (simbolico) è invece tutta una gerarchia. [...] La vita si popola e arricchisce di eventi insostituibili che, appunto perché accaduti una volta per tutte e sovrastanti alle leggi del mondo sublunare, valgono come moduli supremi della realtà, come suo contenuto, significato e midollo, e tutte le

2 È un passo de La terra e la morte (data di composizione l'ottobre 1945), in cui la donna viene identificata con gli elementi del paesaggio: «Anche tu sei collina... Sei la vigna... Sei la terra e la vigna» Pavese (1973c: 57-58). 
vicende quotidiane acquistano senso e valore in quanto ne sono la ripetizione o il riflesso. Un mito è sempre simbolico; per questo non ha mai un significato univoco, allegorico, ma vive di una vita incapsulata che, a seconda del terreno e dell'umore che l'avvolge può esplodere nelle più diverse e molteplici fioriture. Esso è un evento unico, assoluto; un concentrato di potenza vitale da altre sfere che non la nostra quotidiana, e come tale versa un'aura di miracolo in tutto ciò che lo presuppone e gli somiglia. Altra definizione non si può dare del simbolo se non che anch'esso è un oggetto, una qualità, un evento che un valore unico, assoluto, strappa alla causalità naturalistica e isola in mezzo alla realtà. Il più semplice dei simboli, un fazzoletto che l'innamorato ha avuto in dono dalla bella, è tale in quanto ha acquistato un valore assoluto che lo carica di significati molteplici, e questi durano finché dura l'esaltazione amorosa (Pavese 1974: pp. 139-141)³.

Notiamo subito la netta distinzione tra simbolo e allegoria, che continua a mettere in corso le concezioni idealistiche e crociane (Benjamin non è ancora entrato nel patrimonio della casa editrice Einaudi). Si tratta, semmai, di distinguere tra due tipi di simbolo, uno dinamico e uno statico, come troviamo scritto qualche tempo prima nel diario, in una annotazione del 10 dicembre 1939: «Non più simbolo allegorico, ma simbolo immaginoso - un mezzo di più per esprimere la "fantasia" (il racconto). Di qui il carattere dinamico di questi simboli; epiteti che ricompaiono nel racconto e ne sono persone e s'aggiungono alla piena materialità del discorso; non sostituzioni che spogliano la realtà di ogni sangue e respiro, come il simbolo statico (la Prudenza, donna con tre occhi)» (Pavese 1970: 156-157).

Riprenderò poi questo punto; per intanto, notiamo che la questione del simbolo si complica, perché accanto e prima del simbolo c'è il mito:

Il mito è insomma una norma, lo schema di un fatto avvenuto una volta per tutte, e trae il suo valore da questa unicità assoluta che lo solleva fuori del tempo e lo consacra a rivelazione. Per questo esso avviene sempre alle origini, come nell'infanzia: è fuori del tempo. [...] Genuinamente mitico è un evento che come fuori del tempo così si compie fuori dello spazio (Pavese 1974: 140).

È vero, abbiamo sentito che il «mito è sempre simbolico», però il mito comporta un processo narrativo, sia pur elementare, che coincide perfettamente con l'illuminazione puntuale del simbolo (quel suo presentarsi miracolosamente e immediatamente come «un fulmine a ciel sereno»). Il mito prosaicizza. Mentre il simbolo romantico-idealista era infatti realizzato pienamente nella elevazione lirica, per Pavese, invece, il simbolo viene collegato al mito, quindi a un racconto, ad una narrazione più o meno sviluppata.

L'espressione del simbolo-mito non è semplice, anche perché non è chiaro quale sia la struttura profonda e quale quella superficiale: se il mito sia l'esplicazione del simbolo (l'estensione sintattica di un nucleo simbolico), oppure se il simbolo sia l'effetto (il segnale) di uno schema mitico. Comunque sia, Pavese mette in pratica

\footnotetext{
${ }^{3}$ Il saggio è datato 1943-44.
} 
la sua teoria nella stessa Feria d'agosto, in particolare nel brano La vigna. E qui questo luogo deputato, (questo «matrimonio» davvero particolare, diremmo, ricordando Atlantic Oil), è il luogo dell'epifania del senso:

Una vigna che sale sul dorso di un colle fino a incidersi nel cielo, è una vista familiare, eppure le cortine dei filari semplici e profonde appaiono una porta magica. Sotto le viti è terra rossa dissodata, le foglie nascondono tesori, e di là dalle foglie sta il cielo. [...] Tutto ciò è familiare e remoto - infantile, a dirla breve, ma scuote ogni volta, quasi fosse un mondo.

La visione s'accompagna al sospetto che queste non siano se non le quinte di una scena favolosa in attesa di un evento che né il ricordo né la fantasia conoscono. Qualcosa d'inaudito è accaduto o accadrà su questo teatro. [...]

L'uomo sa queste cose contemplando la vigna. E tutto l'accumulo, la lenta ricchezza di ricordi d'ogni sorta, non è nulla di fronte alla certezza di quest'estasi immemoriale. Ci sono cieli e piante, e stagioni e ritorni, ritrovamenti e dolcezze, ma questo è soltanto passato che la vita riplasma come giochi di nubi. La vigna è fatta anche di questo, un miele dell'anima, e qualcosa nel suo orizzonte apre plausibili vedute di nostalgia e di speranza. Insoliti eventi vi possono accadere che la sola fantasia suscita, ma non l'evento che soggiace a tutti quanti e tutti abolisce: la scomparsa del tempo. Questo non accade, è; anzi è la vigna stessa.

Davanti al sentiero che sale all'orizzonte, l'uomo non ritorna ragazzo: è ragazzo. Per un attimo, in cui giunge a far tacere ogni ricordo, si trova entro gli occhi la vigna immobile, istintiva, immutabile, quale ha sempre saputo di avere nel cuore. E non accade nulla, perché nulla può accadere che sia più vasto di questa presenza. Non occorre nemmeno fermarsi davanti alla vigna e riconoscerne i tratti familiari e inauditi. Basta l'attimo dell'incontro e già il ragazzo e l'uomo adulto han cominciato il loro dialogo che, ricco di giorni, dall'inizio non muta (Pavese 1974: 154-155).

Vi sono nel brano due caratteri evidentissimi. Il primo è il carattere soggettivo, personale: è per un particolare soggetto che quel luogo diventa un «luogo unico», sacro e rappresentativo. Il simbolo è anche ricordo dell'infanzia e fusione con essa (il dialogo è interiore: è tra l'adulto e il ragazzo che l'adulto è stato e che può essere ancora grazie al ricordo-simbolo). Il secondo aspetto è l'estaticità: nel brano tutto è fermo, incantato, immemore. Tanto che qui Pavese, per dare voce al simbolo appare costretto a bloccare il racconto, può solo mostrare una scena immobile (se tutto continua ad accadere, allora è lo stesso dire che nulla accade); risultato, qui si ottiene una misura di prosa molto breve, una "pagina" lirica, molto vicina alla famigerata prosa d'arte.

È chiaro che questa soluzione va a confliggere sia con l'imperativo etico del realismo, sia con la possibilità di investigare le valenze collettive del mito, magari sulle tracce della versione archetipica della psicoanalisi, oppure di una concezione socioetnologica del folclore. Ė proprio da questa necessità di approfondimento, dall'esigenza di scavare il problema e di andare alla radice, che nascono e si sviluppano i Dialoghi con Leuco. I Dialoghi costituiscono una svolta nell'opera pavesiana, ma nello stesso tempo rimangono un'intercapedine, un unicum, uno stranissimo prodotto, che nel mentre sembra costituire la fine del viaggio, il gesto coraggioso di 
portare alla luce il mito come tale e di guardarlo in faccia, al contempo costituiscono una complicazione e messa a repentaglio della piena riuscita del gesto miticosimbolico. E non parlo soltanto del loro apparire in controtempo: infatti, nel 1946-47, quando tutti erano impegnati nel rielaborare un passato prossimo sicuramente drammatico, a portare testimonianze di vicende in cui gli scrittori stessi erano stati protagonisti, come si fa a mettersi a riscrivere la mitologia classica? È una scelta provocatoriamente irrealistica, antistorica, non poco aristocratica, e Pavese non può non registrarne gli effetti, nel verificare la scarsa udienza del suo libro. Dalle lettere: i dialoghi «non piacciono a nessuno» (1947); «Leucò è un maledetto libro su cui nessuno osa pronunciarsi» (1947); «sembra impossibile che Leucò non si capisca» (1948) (Pavese 1968, II: 566, 567, 619).

Nello stesso tempo, però, lo attestano le stesse lettere, quest'operina anomala ha per lui un valore molto alto, pressoché decisivo: «Leucò è il mio biglietto da visita presso i posteri» (1950); «un libro che nessuno legge e, naturalmente, è l'unico che vale qualcosa, Dialoghi con Leucò» (1950) (Pavese 1968, II: 753, 769). E ancora nell'intervista alla radio del 1950: «quei dialoghi che sono forse la cosa meno infelice ch'io abbia messo sulla carta [...] Pavese, con ragione, ritiene i Dialoghi con Leucò il suo libro più significativo» (Pavese 1971: 287-289) ${ }^{4}$.

Forse questo strano frutto è solo il portato di una diversione esistenziale: uno strano periodo, il periodo romano e l'incontro con Bianca Garufi, dal cui nome deriva il «biancore» di Leucò. Ma qualunque ne sia la genesi, fatto sta che Pavese scrive quasi insieme ai Dialoghi anche Il compagno, cioè il suo romanzo che maggiormente concede alla prospettiva dell'impegno politico e quindi al superamento dello strato irrazionale verso la consapevolezza e la solidarietà collettiva. Leucò e Compagno costituirebbero, allora, i due estremi divaricati del Logos e del Mythos, provvisoriamente disgiunti e radicalizzati, in attesa di essere di nuovo riunificati nei testi successivi (per i quali l'autore stesso in un appunto userà la formula conciliativa del «realismo simbolico») $)^{5}$.

Ma fino a che punto i Dialoghi segnano il trionfo del mito? Apparentemente, certo, essi lo mettono direttamente in primo piano nella sua forma primordiale e sembrerebbero così dimostrarne la non diminuita attualità, come se non ci fosse niente di nuovo sotto il sole (inutile parlare di partigiani, parlare di Bellerofonte uccisore di mostri è lo stesso, anzi meglio). Ed è altrettanto evidente che la riscrittura del mito antico favorisce la ri-presentazione dei temi cari a Pavese, quelli più irrazionalisti e pessimisti (più pessimisticamente irrazionalisti), quale il ritorno alla terra come regresso mortale e sacrificio rituale: «C'è soltanto la terra, la Madre, la Grotta, che attende sempre e si riscuote soltanto sotto il fiotto di sangue» (L'ospite) (Pavese 1973a: 89), la sessualità come forza incontrollabile («Non c'è dio sopra il sesso... C'è in esso la vita e la

4 E si può aggiungere questa testimonianza di Davide Lajolo: «[Dialoghi con Leucò] È l'unico libro, tra quelli scritti, per il quale ogni giorno si recava all'ufficio diffusione per conoscere come procedeva la vendita» Lajolo (1967: 320-321).

${ }^{5}$ In un appunto dell'ultima parte del diario (1949), Pavese disegna uno specchietto della propria opera in cui Dialoghi con Leucò e Il compagno ricevono a margine l'indicazione «gli estremi: naturalismo e simbolo staccati»; mentre i successivi romanzi da La casa in collina a La luna e i falò sono accompagnati dalla formula fusionale di «realtà simbolica» Pavese (1970: 357). 
morte» (I ciechi) (Pavese 1973a: 22), la donna come nemico feroce e bestiale («questa persona è la belva, la cosa selvaggia, la natura intoccabile» (La belva) (Pavese 1973a: 40) il sacro come fissazione del destino umano e il destino come potere superiore alla volontà e alla storia («nessuno può sfuggire al destino» (La madre); e «Ciò che è stato sarà», (L'inconsolabile) (Pavese 1973a: 54, 77). Tuttavia non mi sembrano indifferenti le modalità scelte da Pavese per riformulare $\mathrm{i}$ suoi temi specifici. Insomma, ancora una volta il contenuto non basta, occorre interrogarsi sulla forma, o per dir meglio, sulla "ideologia della forma": e su questo piano subito si scorgono alcune complicazioni non di poco conto, che forse spiegano l'unicità dei Dialoghi, e che - se non vedo male - ne fanno l'opera pavesiana paradossalmente più versata verso la modernità. Vorrei soffermarmi su tre punti problematici, che chiamerò 1 . la riscrittura; 2. il dialogato; 3. il fermo-immagine.

1. La riscrittura. Secondo la concezione pavesiana enunciata in Feria d'agosto, il mito costituirebbe un modello sottotraccia. Sarebbe una matrice sulla cui base si scrivono, senza saperlo, tutte le storie. Qualunque storia avrebbe dentro di sé il seme del mito. In questo senso, il mito non dovrebbe essere esposto, non ce ne sarebbe alcun bisogno. Tornando ad estrarre il mito e a metterlo in risalto, Pavese si trova costretto a fare i conti con un materiale culturale acquisito nella tradizione e in qualche modo neutralizzato in essa. Riscrivendo la mitologia antica compie un gesto doppiamente contraddittorio verso la sua stessa concezione del mito, perché da un lato lo prende nella sua consistenza non soggettiva ma oggettiva e collettiva, dall'altro lo mette in evidenza invece di lasciarlo estrinsecarsi occultamente. Questa ripetizione non può più essere inconsapevole, diventa una riscrittura progettata che attualizza il mito di proposito: ma allora il rapporto con il materiale di partenza non può non contenere la percezione della distanza "storica". Di qui un certo smagamento critico, del resto indicato apertamente nei trafiletti introduttivi ai dialoghi. Né potrebbe andare altrimenti, perché il mito viene riproposto in un contesto completamente diverso dal suo originario, nel quale era comunitariamente accettato, adesso è in un contesto ormai di increduli, dove appare con la patina blasonata ma polverosa del classico. Pavese neoclassico? Evidentemente no, anzi, semmai "neobarbaro". Intanto bisogna dire che, per quanto la sua riscrittura non sia affatto parodica, ma grave e sofferta (il gioco postmoderno è ancora di là da venire), tuttavia non manca di intervenirvi una quota di ironia. È vero che Pavese non è Savinio che, in quegli stessi anni, fa riemergere il pantheon dell'Olimpo sotto la sferza dell'umorismo, ossia con tutto lo stridore tra il divino-magico e il pratico-borghese che ormai domina il mondo. In Pavese, il ritorno degli dei è del tutto serio. E tuttavia non privo di ironia, un'ironia nera, un'ironia leopardiana, forse, e infatti ho intitolato questo scritto alle «operette morali». ${ }^{6}$

${ }^{6}$ Il nome di Leopardi circola nei Dialoghi con Leucò, nella nota de Il fiore: «i leopardiani Eros e Tànatos» (Pavese 1973a: 32). Lo spunto critico è di Contini, che propone il paragone con qualche cautela quando definisce i Dialoghi «per buona parte almeno poemetti in prosa di forte carica ritmica (...) che non sarebbe improprio considerare un poco la Vita Nuova e le Operette morali del neorealismo» (Contini 1974, IV: 403). Lajolo tuttavia riporta il parallelo all'autore stesso: «Pavese nel mandarmi i Dialoghi con Leucò mi scrisse che, fatte le debite proporzioni, con quel libro, aveva voluto tentare le sue "Operette morali"» (Lajolo 1967: 323). 
Non è un caso, mi sembra, che l'ironia circoli all'inizio. All'inizio, intendo: soprattutto nei primi Dialoghi che Pavese scrisse, ad esempio quello di Circe, dove la prescienza divina inserisce una distanza straniante dagli avvenimenti e lo stesso testo dell'Odissea viene rievocato come se fosse una parte in commedia («gli abbracciai le ginocchia e cominciai la mia battuta: "Chi sei tu? da quale terra generato..." (Le streghe) (Pavese 1973a: 113), e dove quindi l'ironia stessa è dono e appannaggio degli dei, contrapposta alla vocazione degli umani per la ricerca del senso fino alla tragedia: «Sì. Qualcuno di loro sa ridere davanti al destino, sa ridere dopo, ma durante bisogna che faccia sul serio o che muoia. Non sanno scherzare sulle cose divine, non sanno sentirsi recitare come noi» (Pavese 1973a: 113). Ironia nell'inizio e negli inizi, intendo: perché l'ironia continua a circolare soprattutto nei brevi brani di apertura (che sono poi gli unici sia pur rapidamente narrativi) dove si rimanda a un sapere condiviso, ma si sottolineano anche le lacune e le crepe del materiale tratto da Omero, dai tragici o da altrove. Può esserci, ad esempio, una negazione critica «Che Issione finisse nel Tartaro per la sua audacia, è probabile. Falso invece che generasse i Centauri dalle nuvole» (Pavese 1973a: 8), una sfumatura autoironica: «Superfluo rifare Omero» (Pavese 1973a: 58), l'indicazione del riempimento di una lacuna: «Licaone, signore d'Arcadia, per la sua inumanità venne mutato in lupo da Zeus. Ma il mito non ci dice dove e come sia morto» (Pavese 1973a: 82), una correzione della fonte: «Di ciò Omero non ha tenuto quel conto che si vorrebbe» (Pavese 1973a: 112), oppure ancora l'uso dell'eufemismo: «Sono noti i luttuosi incidenti che hanno funestato la casa degli Atridi» (Pavese 1973a: 126); tutte forme che sottolineano l'intervento di manipolazione su un testo-base (l'ipotesto della riscrittura) che non sembra affatto in grado di «parlare da solo» come al mito converrebbe, se ha bisogno di simili note di accompagnamento e di verifica.

2. Il dialogato. Perché Pavese non ha riscritto il mito narrativamente ma gli ha dato forma di dialogo? Una risposta potrebbe essere: in ossequio al genere-maestro della tragedia, per il fatto che il suo stesso ritorno al mito aveva, inesorabilmente, un valore tragico. Questo potrebbe essere avvalorato dalla presenza di alcuni eroi sommamente tragici (Edipo in primis), nonché dal fatto che la tattica del dialogo sorge da uno squilibrio di base tra un personaggio protagonista e un personaggio «di spalla» che lo spinge a manifestarsi e a giustificarsi nella propria difficoltà e spesso nel proprio fallimento. E tuttavia, a guardar bene, il ruolo del secondo agonista non è affatto di supporto. La duplicità del dialogo corrisponde a una polarità dialettica di contrasti assolutamente decisivi. Tanto che, si potrebbe affermare che il senso dei Dialoghi come espressione dei conflitti interni di Pavese, sta esattamente in una trafila di opposizioni: dei/uomini; natura/cultura; destino/volontà; istinto/morale; sapere/conoscenza. In sintesi inconscio/ragione, oppure, tornando al dunque, mythos/logos. Più ancora o in più al contrasto tra dei e uomini è il contrasto (fin dal Dialogo posto in apertura) tra due tipi di divino, il titanico e l'olimpico: un divino primordiale legato all'identificazione «selvaggia» con la natura, e un nuovo livello religioso più astratto, fondato sulla «legge», e di conseguenza sulla divisione dei livelli e dei ruoli. Dopo il taglio della divisione, il ritorno al prima è impossibile: il prima, però, continua ad angustiare con il suo inevitabile ritorno e la sua minaccia di ricaduta. 
Il dialogismo (e non è strano, pensiamo a Bachtin) è la matrice profonda della vocazione narrativa pavesiana, come attesta molto presto il diario, «La tua vera musa prosastica è il dialogo» (Pavese 1970: 67) ${ }^{7} ; 22$ dicembre 1937). Ma, nell'affidarsi al dialogo come genere, Pavese compie un passo in più, con alcune conseguenze di rilievo: i personaggi dei Dialoghi diventano portatori di polarità e di astrazioni, il grado della loro rappresentanza aumenta, si spogliano della personalità simil-umana per indicare porzioni, propensioni, tensioni interne alla personalità e all'identità individuale; sicché malgrado la posizione teorica favorevole al simbolo, il testo pavesiano finirebbe per andare a promuovere un tipo di organizzazione incline proprio verso la tanto avversata allegoria ${ }^{8}$. Per altro, mentre il ritorno al mito dovrebbe svolgersi nell'atmosfera extrastorica del passato classico, si può vedere insorgere, per forza di cose, l'allegoria dell'attualità. Non è soltanto il parallelo tra dei e padroni o l'evocazione del comunismo ( «Vedi, gli dèi sono i padroni. Sono come i padroni» e subito dopo: «quante case di padroni bisogna incendiare, quanti ammazzarne per le strade e per le piazze, prima che il mondo torni giusto e noi si possa dir la nostra?» (Pavese 1973a: 97); più chiaro di così...). In fondo tutto il sapere ancestrale non è che l'avvertimento di una base agricola (qui alle influenze di Vico, Jung, Frazer, si somma quella di De Martino), che non può essere superata per semplice colonizzazione da parte della coscienza di classe proletaria. La resistenza della cultura contadina - una questione che oggi appare lontana, ma che forse non ha smesso di rimanere sottotraccia in molti fenomeni di rifeudalizzazione, - questo che potremmo chiamare il "ritorno del superato", è la molla di un contrasto dialogico-dialettico senza soluzione.

3. Il fermo-immagine. Mi pare la questione più interessante. Infatti il mito è propriamente racconto e sviluppo narrativo. Qui invece Pavese lo coglie in un punto preciso e lì lo blocca con l'alterno discutere surplace del dialogo. Questo nucleo narrativo fermato e distribuito tra le voci dialoganti finisce per assomigliare molto a quella che Benjamin chiamava «l'inquietudine irrigidita», facendone uno dei caratteri basilari della sua concezione dell'allegoria. Di nuovo un racconto immobile, ma questa volta non risolto nell'estasi, ma in uno scambio conflittuale. In parte lo stesso Pavese si rendeva conto di come l'esplicitazione del mito lo portasse a compiere una svolta contraria al romanzo (lo attesta una lettera dell'agosto 1947: si va «svogliando» del genere ${ }^{9}$ ). È probabile che poi lui stesso abbia preferito lasciare l'esperimento come unicum, e abbia preferito tornare all'ovile, anche a causa delle reazioni dell'ambiente letterario, e tuttavia questo libretto fuori-genere oltre che fuori-

${ }^{7}$ Per l'uso del dialogato e della battuta, si veda lo studio di Mutterle (1977), in particolare il capitolo «La lingua witty».

${ }^{8}$ Tra le altre cose, nel diario, troviamo anche una indicazione che non sembrerebbe del tutto avversa all'allegoria: «Allegoria è ogni simbolo visto attraverso l'intelligenza» (Pavese 1970: 306); positivo o negativo a seconda della sfumatura che si conferisce alla parola «intelligenza».

${ }^{9}$ Lettera a Pancrazi: «non credo che potrò attuare il suo consiglio di costruire una grossa e più impersonale macchina narrativa; anzi mi vado svogliando di questo genere e, a dirla schietta, non ci credo più gran che. Sta anzi per uscire qualcosa [è appunto Leucò] - che le manderò - da cui vedrà quanto mi sono andato corrompendo» (Pavese 1968, II: 551). 
tempo resta a far parte di una storia della scrittura novecentesca di prosa anti-narrativa, ancora tutta da scrivere.

E, anche a non voler calcare la cosa fino a questo punto, certo consapevolmente Pavese ha costruito la prosa dei Dialoghi con una andatura cadenzata non lontana dalla metrica anapestica adottata nella poesia. Insomma, prima era poesia che andava verso la prosa, mentre chiasticamente, adesso, è prosa che va verso la poesia. Un esempio, tra i mille possibili:

Nella mia patria si racconta che sull'Ida nascevano dèi. Nessun mortale è mai salito oltre gli ultimi boschi. Noi temiamo anche l'ombra che cade dal monte. Come posso accettare le cose che dici? (Pavese 1973a: 150).

Con qualche scivolamento dal ritmo ternario a quello quaternario è tuttavia sempre una battuta ben ritmata a reggere il "passo" del discorso. Se è vero che la cadenza è un diretto portato della materia mitica, in quanto la ripetizione ossessiva è precisamente nella natura del mito, ${ }^{10}$ tuttavia lo spessore che ne ricava la scrittura in prosa produce varie conseguenze. La scrittura ne viene posta in evidenza, e ne riceve uno stigma inequivocabilmente innaturale e perciò antinaturalistico. Nessuno parlerebbe così. La rappresentazione perde il carattere mimetico-sociologico, per mostrare il proprio carattere di costruzione conoscitiva. Ma, nella tradizione romantico-idealista, il simbolo avrebbe dovuto garantire la trasparenza (non dovrebbe farsi vedere e intralciare la superficie dell'opera) e ciò faceva sì che addirittura in Lukács potesse diventare un alleato del realismo. Pavese, nel diario, insiste con il dire che con i Dialoghi ha scoperto e sperimentato «una nuova forma» ${ }^{11}$. Ma l'insistenza sulla forma non è senza rischi; la eccessiva visibilità della forma (in questo caso la rilevanza del ritmo ribattuto) è un altro punto d'inciampo per l'opera usualmente istantanea e inconsapevole del simbolo.

Potremo prendere, per un'ultima e rapidissima verifica, il dialogo intitolato $L a$ vigna. Un'altra vigna, di nuovo ${ }^{12}$. Con qualche parentela con quella di Feria d'agosto, anche questa immersa nell'aura del simbolo, nella piuttosto dannunziana comunione con la natura: «Tu sei mai stata in un vigneto in costa a un colle lungo il mare, nell'ora lenta che la terra dà il suo odore? Un odore rasposo e tenace, tra di fico e di pino? Quando l'uva matura, e l'aria pesa di mosto? O hai mai guardato un melograno, frutto e fiore? Qui regna Dioniso, e nel fresco dell'edera, nei pineti e sulle aie» (Pavese 1973a: 140-1). Se volessimo andare a vedere la sorte del binomio

\footnotetext{
10 Scrive Pavese in Raccontare è monotono (1949): «dire stile è dire cadenza, ritmo, ritorno ossessivo del gesto e della voce, della propria posizione entro la realtà» (Pavese 1971: 332).

11 Gli appunti in proposito sono almeno due: una in sede di bilancio del 1945, «scoperta una nuova forma che sintetizza molti filoni (il dialogo di Circe)»; un'altra più avanti nel '46, sul "contagio della forma", «Per chi sa scrivere, una forma è sempre qualcosa d'irresistibile. Corre il rischio di dire sciocchezze e di dirla male, ma la forma che lo tenta, pronta ad imbeversi delle sue parole, è irresistibile. (Intendo per esempio il genere del dialoghetto mitologico tuo)» Pavese (1970: 304).

12 Vigne su vigne. Un'altra vigna epifanica si trova in un racconto, dello stesso periodo dei Dialoghi, inserito in Feria d'agosto, e intitolato Storia segreta. A proposito di questo tema insistito, si veda la voce «Vigna» nella monografia-repertorio di Gigliucci (2001: 193-196).
} 
metafora e metonimia, potremmo scoprire che la metonimia pertiene al mondo della prassi umana, contraddistinto dalla separazione, dall'abbandono e dalla solitudine (Teseo, che ha lasciato Ariadne, non è mai nominato direttamente, ma è indicato per metonimia, la "nave" o per sineddoche della metonimia, «la vela nera»); mentre la metafora accompagna lo sfogo del dolore («finché il cuore non ti scoppierà, finché non latrerai come una cagna e vorrai spegnerti nel mare come un tizzo»; qui sono ancora similitudini) e soprattutto l'avvento del sacro, che promette una nuova fusione «oltreumana» («gli dèi sono il luogo... Verrà Dioniso e ti parrà di essere rapita da un gran vento»).

Ma dovremmo anche aggiungere qualcosa sul lato negativo della metafora che, giunta all'apice rivelativo, rischia di ribaltarsi nella distruzione. «Tutti gli dei sono crudeli», dice il dialogo, la fusione è la morte. Ciò è evidenziato dalla presenza rilevante dell'ossimoro: il dio «uccide ridendo»; "per svegliarti più forte, devi cedere al sonno». E l'ossimoro - che cancella con il suo «stato di stallo» il fiorire metaforico - indica, appunto, il prevalere dell'ambivalenza e della contraddizione ${ }^{13}$.

Insomma, ho provato a decostruire Pavese: partito dalla volontà di ispezionare la «base» mitico-simbolica, di tirarla in piena luce e di esporla nella sua versione più antica e perciò si presume più autentica, l'autore si è trovato in mano invece di una Verità con la maiuscola sussistente $a b$ aeterno, diverse entità non solo multiple polimorfe e proteiformi, ma discordanti e contrastanti, in un puzzle incomponibile.

Giustamente la Muñiz Muñiz ha parlato di fallimento per i Dialoghi con Leuco ${ }^{14}$, nel senso che non è possibile estrarre da essi alcuna tesi dimostrativa ben risolta. Alle somme non sono né completamente razionalistici-ironici (il mito ritornerebbe per essere dissezionato, riportato alle sue radici storiche, demistificato senza appello), ma neppure interamente irrazionalistici-estetici (qualunque cosa faccia l'uomo civilizzato è poi sempre il selvaggio che riappare sotto la sottile crosta della cultura).

Basta vedere come si pone il problema del «risveglio» (benjaminiano quant'altri mai): «temo il risveglio, come tu temi la morte»; e, lo abbiamo visto, l'inversione del passaggio attraverso l'irrazionale, "per svegliarti più forte, devi cedere al sonno». La strategia di Pavese sembra andare al contrario del risveglio, immergersi nel «gorgo» dell'incoscienza, della ripetizione fatale - «destino», «sorte» sono termini-refrain che tornano ad ogni piè sospinto -; tuttavia, nello stesso tempo, è altrettanto costretta alla ricerca di una lucida (stoica) resistenza ad occhi aperti, senza la quale non sarebbe concepibile neppure l'opera intrapresa dallo scrittore. La metafora del «gorgo» si declina così, per gli scrittori: «un tentativo di vedere, nel gorgo del

13 Sulla «contraddizione» pavesiana si è soffermata la critica più avvertita. Guido Guglielmi ha posto il contrasto tra mito e logos come antagonismo inconciliabile: «La drammatica contraddizione di Pavese era così inevitabile (...). Sicché la sua scrittura appare naturalistica e simbolica a un tempo, cioè internamente squilibrata» in Guglielmi (1967: 146). Anche Ettore Catalano sottolinea «la struttura costitutivamente contraddittoria dei Dialoghi» Catalano (1976: 113). E Mutterle sostiene che Pavese «del contraddittorio si fa una ragion d'essere e di stile» Mutterle (1977: 86).

14 Anche per quanto riguarda il rapporto tra simbolo e allegoria: «Tale groviglio non poteva che rendere complementari simbolo e allegoria (mistero e chiarezza razionale) generando così un circolo vizioso anziché una sintesi dialettica» Muñiz Muñiz (1992: 130).

15 Ma il «gorgo» si declinerà anche così: «scenderemo nel gorgo muti» Pavese (1973c: 77). 
mito che li afferra, il più nitidamente possibile» (Pavese 1971: 299) ${ }^{15}$. Riscrivere il mito antico per andare alla radice, richiede di «estrarre» precisamente il modello e di esporlo, invece di lasciarlo estrinsecarsi di nascosto, inconsciamente.

La non conclusione e non conciliazione delle due tensioni, spinge alla dialogizzazione e dialettizzazione del testo, tra coazione e volontà, ripetizione e applicazione attualizzante. Da cui si generano le diverse e diffuse asimmetrie tecniche del dialogo, dai dialoghi in cui gli interlocutori parlano di un eroe assente a quelli in cui si parla a un morto, ecc. Insomma, proprio su quello che avrebbe dovuto essere il suo terreno, il simbolo incontra difficoltà: tolto dall'illuminazione paesaggistica e portato nell'ambito della dialettica culturale e collettiva, si complica e si corrode. La stessa critica della modernità, avanzata dall'autore con la sfiducia nella ragione e la necessità del suo confronto disperato, fuori di ogni trionfalismo storicista, con una base immutabile e immodificabile, si ingolfa in uno scontro irrisolto che, irradiandosi nelle piegature e nei decalages testuali, in virtù dei paradossi della scrittura, perviene ad una rappresentazione solcata dalla crisi e dalla conclusione aperta, cioè quella più propria della modernità.

\section{Bibliografia}

Catalano, Ettore (1976): Cesare Pavese fra politica e ideologia. Bari, De Donato.

ContinI, Gian Franco (1974): La letteratura italiana, tomo IV. Firenze-Milano, Sansoni Accademia.

GigliUCCI, Roberto (2001): Cesare Pavese. Milano, Mondadori.

GUGLIELMI, Guido (1967): Letteratura come sistema e come funzione. Torino, Einaudi. LAJOLO, Davide (1967): Il vizio assurdo. Milano, Il Saggiatore.

MuÑIZ MuÑIZ, Ma de las Nieves (1992): Introduzione a Pavese. Bari, Laterza.

MUTTERLE, Anco M. (1977): L'immagine arguta. Torino, Einaudi.

PAVESE, Cesare (1968): Lettere (1926-1950), a cura di Italo Calvino. Torino, Einaudi.

PAVESE, Cesare (1970): Il mestiere di vivere. Torino, Einaudi.

PAVESE, Cesare (1971): La letteratura americana e altri saggi. Milano, Il Saggiatore.

PAVESE, Cesare (1973a): Dialoghi con Leucò. Torino, Einaudi.

PAVESE, Cesare (1973b): Lavorare stanca. Torino, Einaudi.

PAVESE, Cesare (1973c): Le poesie del disamore. Torino, Einaudi.

PAVESE, Cesare (1974): Feria d'agosto. Torino, Einaudi. 